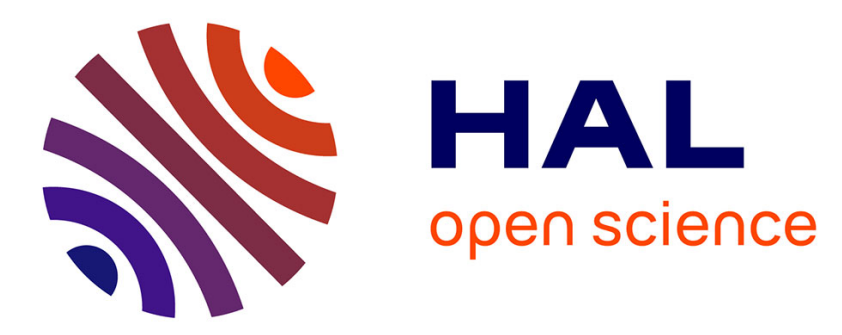

\title{
Workspace and joint space analysis of the 3-RPS parallel robot
}

\author{
Damien Chablat, Ranjan Jha, Fabrice Rouillier, Guillaume Moroz
}

\section{To cite this version:}

Damien Chablat, Ranjan Jha, Fabrice Rouillier, Guillaume Moroz. Workspace and joint space analysis of the 3-RPS parallel robot. ASME 2013 International Design Engineering Technical Conferences \& Computers and Information in Engineering Conference, Aug 2014, Buffalo, United States. pp.1-10. hal-01006614

\section{HAL Id: hal-01006614 https://hal.science/hal-01006614}

Submitted on 16 Jun 2014

HAL is a multi-disciplinary open access archive for the deposit and dissemination of scientific research documents, whether they are published or not. The documents may come from teaching and research institutions in France or abroad, or from public or private research centers.
L'archive ouverte pluridisciplinaire HAL, est destinée au dépôt et à la diffusion de documents scientifiques de niveau recherche, publiés ou non, émanant des établissements d'enseignement et de recherche français ou étrangers, des laboratoires publics ou privés. 


\section{Proceedings of the ASME 2014 International Design Engineering Technical Conferences \& Computers and Information in Engineering Conference IDETC/CIE 2014 \\ August 17-20, 2014, Buffalo, USA}

\section{DETC2014/MR-34593}

\section{WORKSPACE AND JOINT SPACE ANALYSIS OF THE 3-RPS PARALLEL ROBOT}

\author{
D. Chablat, R. Jha \\ Institut de Recherche en Communications \\ et Cybernétique de Nantes \\ (UMR CNRS 6597) France \\ Email addresses: \\ Damien.Chablat@irccyn.ec-nantes.fr \\ Ranjan.Jha@irccyn.ec-nantes.fr
}

\author{
F. Rouillier \\ INRIA Paris-Rocquencourt \\ Institut de Mathématiques de Jussieu \\ (UMR CNRS 7586), France \\ Email addresses: \\ Fabrice.Rouillier@inria.fr
}

\author{
G. Moroz \\ INRIA Nancy-Grand Est, \\ France \\ Email addresses: \\ Guillaume.Moroz@inria.fr
}

\begin{abstract}
The Accurate calculation of the workspace and joint space for 3 RPS parallel robotic manipulator is a highly addressed research work across the world. Researchers have proposed a variety of methods to calculate these parameters. In the present context a cylindrical algebraic decomposition based method is proposed to model the workspace and joint space. It is a well know feature that this robot admits two operation modes. We are able to find out the set in the joint space with a constant number of solutions for the direct kinematic problem and the locus of the cusp points for the both operation mode. The characteristic surfaces are also computed to define the uniqueness domains in the workspace. A simple 3-RPS parallel with similar base and mobile platform is used to illustrate this method.
\end{abstract}

\section{INTRODUCTION}

The workspace of parallel robots mainly depends upon the actuated joint variables, the range of motion of the joints and the mechanical interferences between the bodies of mechanism. There are different techniques based on geometric, discretization, numerical and algebraic methods which are used to calculate the workspace of parallel robot. The main advantage of the geometric approach is that, it establish the nature of the boundary of the workspace [1]. Also it allows the computation of the surface and volume of the workspace while being very efficient in terms of storage space, but if the rotational motion is included, it becomes more complex. The interval analysis based method can be used to compute the workspace but the computation time depends on the complexity of the robot and the accuracy requested. The ALIAS library is a good implementation for the parallel robots [2]. Discretization methods are usually less complex and take into account all kinematic constraints, but require more space and computation time for higher resolutions. The majority of numerical methods which is used to determine the workspace of parallel manipulators includes the discretization of the pose parameters for the determination of workspace boundaries [3]. Algebraic methods are used in [4-6] to study planar or spatial parallel robots. Two main teps are necessary to perform the workspace and jointspace analysis. First, the discriminant variety is computed to characterize the boundaries of the workspace and jointspace as well as the singularities. Second, the Cylindrical Algebraic Decomposition (CAD) is used to define the connected regions where there exists a constant number of real solutions to the inverse and direct kinematic problem and no parallel or serial singularities [5-7].

For the design or the trajectory planning, the workspace of the parallel manipulator is divided into singularity-free regions [8]. The singularities divide the workspace into aspects and the characteristic surfaces induce a partition of each aspect into a set of regions (the basic regions) [9]. For the parallel robots with several inverse and direct kinematic solutions, the aspects are de- 
fined as the maximal singularity-free sets in the workspace or the cross-product of the joint space by the workspace. An assembly mode is associated with a solution for the Direct Kinematic Problem (DKP) and a working mode for the Inverse Kinematic Problem (IKP). Practically, a change of assembly mode may occur during the execution of a trajectory between two configurations in the workspace which are not necessarily associated with the same input for a given working mode. The uniqueness domains can be defined as the maximal regions of the workspace where all the displacements of the end-effector can be accomplished without changing of assembly mode and working mode [5]. The main goal of this paper is to generalize the notions of the uniqueness domains for parallel robot with several operation modes and a single working mode.

The outline of the paper is as follows. Firstly, the kinematic equations of the 3-RPS parallel robot under study are introduced. The singular configurations are written for both operation modes. The cylindrical algebraic decomposition algorithm is presented to explain the study of the workspace and the joint space. Then, the characteristic surface is defined for the parallel robots with several operations. Finally, the basic regions and the basic components are computed to define the uniqueness domains for the direct kinematic problem.

\section{KINEMATICS OF 3 RPS}

The robot under study is the 3-RPS parallel robot with three degrees of freedom and has been studied by many researchers $[10,11]$. It is the assembly of two equilateral triangles (the base, moving platform) by three identical RPS legs where $\mathrm{R}$ is a revolute passive joint, $\underline{\mathrm{P}}$ an actuated prismatic joint and $\mathrm{S}$ a passive spherical joint. Thus, the revolute joint is connected to the fixed base and the spherical joint to the mobile platform.

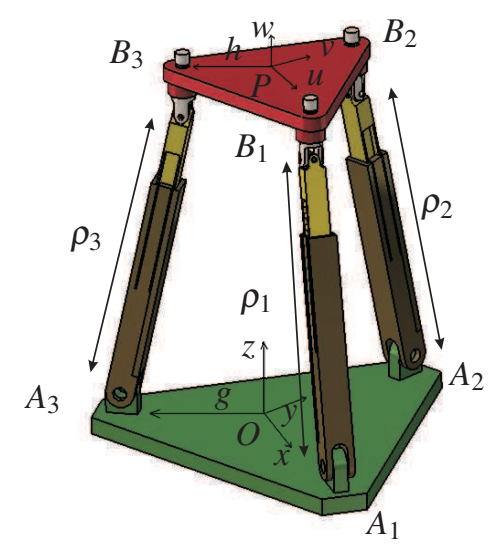

FIGURE 1. 3-RPS parallel robot
Considering the 3-Rㅗ S parallel manipulator, as shown in figure 1 , the fixed base consists of an equilateral triangle with vertices $A_{1}, A_{2}$ and $A_{3}$, and circumradius $g$. The moving platform is another equilateral triangle with vertices $B_{1}, B_{2}$ and $B_{3}$ and circumradius $h$, and circumcenter $P$. The two design parameters $g$ and $h$ are positive numbers. Connecting each of the vertices' pairs $A_{i}, B_{i}(i=1,2,3)$ by a limb, a rotational joint lies at $A_{i}$ and a spherical joint lies at $B_{i} . \rho_{i}$ denotes the length of each limb and the adjustment is done through an actuated prismatic joint. Thus, there are five parameters, namely $g, h, \rho_{1}, \rho_{2}$ and $\rho_{3}$. The $g$ and $h$ parameters determine the design of the manipulator whereas the joint parameters $\rho_{1}, \rho_{2}$ and $\rho_{3}$ determine the motion of the robot. To simplify the equations, we will study a robot with $g=h=1$, which permits to have simple constraint equations.

\section{Kinematic equations}

Spatial rotations in three dimensions can be parametrized using Euler angles [12], unit quaternions [13] or dual quaternions [10]. The quaternion representation is used for modeling the orientation as quaternions do not suffer from singularities as Euler angles do. Moreover, to transform the trigonometric equations to algebraic equations, we may either introduce the singularity of the transformation $t=\tan (\alpha / 2)$ or replace the angle $\alpha$ by two parameters $\cos _{\alpha}$ and $\sin _{\alpha}$ with $\cos _{\alpha}^{2}+\sin _{\alpha}^{2}-1$. In addition, it is easier to represent workspace sections with the quaternions than the dual quaternions.

A quaternion $\mathbf{q}$ is defined by

$$
\mathbf{q}=q_{1}+q_{2} \mathbf{i}+q_{3} \mathbf{j}+q_{4} \mathbf{k}
$$

The quaternion rotation matrix for the parallel robot is then

$$
\mathbf{Q}=\left[\begin{array}{ccc}
2 q_{1}^{2}+2 q_{2}^{2}-1 & -2 q_{1} q_{4}+2 q_{2} q_{3} & 2 q_{1} q_{3}+2 q_{2} q_{4} \\
2 q_{1} q_{4}+2 q_{2} q_{3} & 2 q_{1}^{2}+2 q_{3}^{2}-1 & -2 q_{1} q_{2}+2 q_{3} q_{4} \\
-2 q_{1} q_{3}+2 q_{2} q_{4} & 2 q_{1} q_{2}+2 q_{3} q_{4} & 2 q_{1}^{2}+2 q_{4}^{2}-1
\end{array}\right]
$$

with $q_{1}^{2}+q_{2}^{2}+q_{3}^{2}+q_{4}^{2}=1$. The transformation from the moving frame to the fixed frame can be described by a position vector $\mathbf{p}=O P$ and a $3 \times 3$ rotation matrix $\mathbf{R}$. Let $\mathbf{u}, \mathbf{v}$ and $\mathbf{w}$ be the three unit vectors defined along the axes of the moving frame, then the rotation matrix can be expressed in terms of the coordinates of $\mathbf{u}$, $\mathbf{v}$ and $\mathbf{w}$ as:

$$
\mathbf{R}=\left[\begin{array}{lll}
u_{x} & v_{x} & w_{x} \\
u_{y} & v_{y} & w_{y} \\
u_{z} & v_{z} & w_{z}
\end{array}\right]
$$


The vertices of the base triangle and mobile platform triangle are

$$
\begin{array}{cc}
\mathbf{A}_{1}=\left[\begin{array}{l}
g \\
0 \\
0
\end{array}\right] \quad \mathbf{A}_{2}=\left[\begin{array}{c}
-g / 2 \\
g \sqrt{3} / 2 \\
0
\end{array}\right] \quad \mathbf{A}_{3}=\left[\begin{array}{c}
-g / 2 \\
-g \sqrt{3} / 2 \\
0
\end{array}\right] \\
\mathbf{b}_{1}=\left[\begin{array}{l}
h \\
0 \\
0
\end{array}\right] \quad \mathbf{b}_{2}=\left[\begin{array}{c}
-h / 2 \\
h \sqrt{3} / 2 \\
0
\end{array}\right] \quad \mathbf{b}_{3}=\left[\begin{array}{c}
-h / 2 \\
-h \sqrt{3} / 2 \\
0
\end{array}\right]
\end{array}
$$

The coordinates of $\mathbf{b}_{i}$ with respect to fixed frame reference are obtained by $\mathbf{B}_{i}=\mathbf{P}+\mathbf{R} \mathbf{b}_{i}$ for $i=1,2,3$. Also the coordinates of the centre of the mobile platform in the fixed reference is $\mathbf{P}=$ $\left[\begin{array}{lll}x & y & z\end{array}\right]^{T}$. The distance constraints yields:

$$
\left\|\mathbf{A}_{i}-\mathbf{B}_{i}\right\|=\rho_{i}^{2} \quad \text { with } \quad i=1,2,3
$$

As $A_{i}$ are revolute joints, the motion of the $B_{i}$ are constrained in planes. This leads to the three constraint equations:

$$
\begin{array}{r}
u_{y} h+y=0 \\
y-u_{y} h / 2+\sqrt{3} v_{y} h / 2+\sqrt{3} x-\sqrt{3} u_{x} h / 2+3 v_{x} h / 2=0 \\
y-u_{y} h / 2-\sqrt{3} v_{y} h / 2-\sqrt{3} x+\sqrt{3} u_{x} h / 2+3 v_{x} h / 2=0
\end{array}
$$

Solving with respect to $x$ and $y$ we get:

$$
\begin{aligned}
& y=-h u_{y} \\
& x=h\left(\sqrt{3} u_{x}-\sqrt{3} v_{y}-3 u_{y}+3 v_{x}\right) \sqrt{3} / 6
\end{aligned}
$$

In Equations 6, 8, 9, we substitute $x, y$ using relations 10 and 11, and $\mathbf{u}, \mathbf{v}, \mathbf{w}$ by quaternion expressions using 2 . Then (8) and (9) become $q_{1} q_{4}=0$. Thus, we have either $q_{1}=0$ or $q_{4}=0$. This property is associated with the notion of operation mode. To obtain the algebraic equations, we replace $\sqrt{3}$ by the variable $S_{3}$ and add the equation $S_{3}^{2}-3=0$ and the constraint $s_{3}>0$.

\section{Operation Modes}

Notion of operation mode (OM) was introduced in [14] to explain the behavior of the DYMO robot. An operation mode is associated with a specific type of motion. For the DYMO robot, there are five operation modes: translational, rotational, planar (two types) and mixed motions. In the workspace $W$, for each operation mode, $W_{O M^{j}}$ is defined such that

- $W_{O M^{j}} \subset W$
- $\forall X \in W_{O M^{j}}, \mathrm{OM}$ is constant

For a parallel robot with several operating modes, the pose can be defined by fixing the same number of parameters as the degree of freedom of the mobile platform. Given an operation mode $O M^{j}$, if we have a single inverse kinematic solution, we can define an application that maps $\mathbf{X}$ onto $\mathbf{q}$ :

$$
g_{j}(\mathbf{X})=\mathbf{q}
$$

Then, the images in $W_{O M^{j}}$ of a posture $\mathbf{q}$ in the joint space $Q$ are defined by:

$$
g_{j}^{-1}(\mathbf{q})=\left(\mathbf{X} /(\mathbf{X}, \mathbf{q}) \in O M^{j}\right)
$$

where $g_{j}^{-1}$ stands to be the direct kinematic problem for the operation mode $j$.

\section{Singularities}

Differentiating the constraints equations with respect to time leads to the velocity model:

$$
\mathbf{A t}+\mathbf{B} \dot{\mathbf{q}}=0
$$

where $\mathbf{A}$ and $\mathbf{B}$ are the parallel and serial Jacobian matrices, respectively, $\mathbf{t}$ is the velocity of $P$ and $\dot{\mathbf{q}}$ joint velocities. The parallel singularities occur whenever $\operatorname{det}(\mathbf{A})=0$. Let $O M^{1}$ be the operation mode where $q_{1}=0$ and $O M^{2}$, where $q_{4}=0$, then $\mathscr{S}_{O M^{1}}$ and $\mathscr{S}_{\mathrm{OM}^{2}}$ represent the singularity locus and are characterized by:

$$
\begin{array}{r}
\mathscr{S}_{O M^{1}}: q_{4}\left(8 q_{2} q_{3}^{2} q_{4}^{6}+2 q_{2} q_{4}^{8}-64 z q_{3}^{6} q_{4}-96 z q_{3}^{4} q_{4}^{3}-36 z q_{3}^{2} q_{4}^{5}\right. \\
-6 z q_{4}^{7}-24 z^{2} q_{2} q_{3}^{2} q_{4}^{2}-6 z^{2} q_{2} q_{4}^{4}-32 q_{2} q_{3}^{2} q_{4}^{4}-10 q_{2} q_{4}^{6}+2 z^{3} q_{4}^{3} \\
+96 z q_{3}^{4} q_{4}+72 z q_{3}^{2} q_{4}^{3}+23 z q_{4}^{5}+16 z^{2} q_{2} q_{3}^{2}+10 z^{2} q_{2} q_{4}^{2}+8 q_{2} q_{4}^{4} \\
\left.-z^{3} q_{4}-36 z q_{3}^{2} q_{4}-21 z q_{4}^{3}-4 z^{2} q_{2}+4 z q_{4}\right)=0
\end{array}
$$

$$
\begin{array}{r}
\mathscr{S}_{O M^{2}}: q_{1}^{2}\left(6 q_{1}^{7} q_{3}+8 q_{1}^{5} q_{3}^{3}-2 z q_{1}^{6}+36 z q_{1}^{4} q_{3}^{2}+96 z q_{1}^{2} q_{3}^{4}\right. \\
+64 z q_{3}^{6}-18 z^{2} q_{1}^{3} q_{3}-24 z^{2} q_{1} q_{3}^{3}-18 q_{1}^{5} q_{3}-16 q_{1}^{3} q_{3}^{3}+2 z^{3} q_{1}^{2} \\
+3 z q_{1}^{4}-72 z q_{1}^{2} q_{3}^{2}-96 z q_{3}^{4}+18 z^{2} q_{1} q_{3}+12 q_{1}^{3} q_{3}-z^{3} \\
\left.+3 z q_{1}^{2}+36 z q_{3}^{2}-4 z\right)=0
\end{array}
$$

The serial singularities occur whenever $\operatorname{det}(\mathbf{B})=0$ i.e $\rho_{1} \rho_{2} \rho_{3}=$ 0 . The common coordinates for the both operation modes are $z$, $q_{2}$ and $q_{3}$. Figure 2(a) represents the singularity curve for $O M^{1}$ 


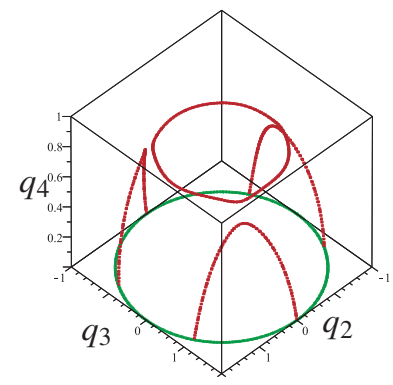

(a)

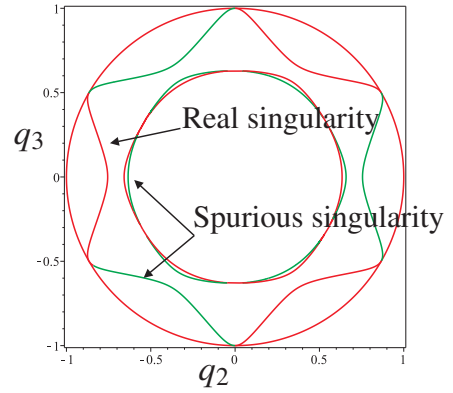

(b)
FIGURE 2. Singularity curves for $z=3, q_{1}=0$ (a) and its 2D projection under $\left(q_{2}, q_{3}\right)$ (b)

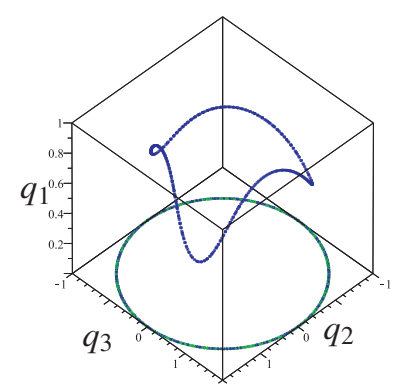

(a)

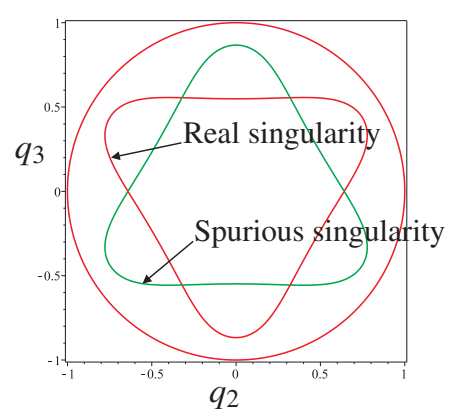

(b)
FIGURE 3. Singularity curves for $z=3, q_{4}=0$ (a) and its 2D projection under $\left(q_{2}, q_{3}\right)$ (b)

for a fixed value of $z$ (i.e $z=3$ ) and Figure 2(b) shows its projection in the two dimensional coordinate space $\left(q_{2}-q_{3}\right)$.

Due to the redundancy of the quaternion representation there exists two triplets based on these three coordinates to represent the same pose in the same operation mode. To overcome this problem, we set $q_{1}>0$ and $q_{4}>0$. We can then depict a slice of this surface by fixing one parameter as shown in Figures 2(a) and 3(a). Figures 2(b) and 3(b) shows the singularity curves in the projection space $z, q_{2}$ and $q_{3}$, where the red curve is a real singular locus, which represents the positive values of $q_{4}$ or $q_{1}$ and the green curve is a spurious singularity curve, which represents the negative values of $q_{4}$ or $q_{1}$.

\section{Cylindrical algebraic decomposition}

The workspace (resp. Joint space) analysis classifies the number of solutions of the parametric system associated with the Inverse (resp. Direct) Kinematic Problem (IKP). This method was introduced for parallel robots in [5]. We will recall here the main steps of the computation and the new step for a robot with several operation modes. For such operations, both workspace as well as joint space is decomposed into cells $C_{1}, \ldots, C_{k}$, such that:
- $C_{i}$ is an open connected subset of the workspace;

- for all pose values in $C_{i}$, the direct (resp. inverse) kinematics problem has a constant number of solutions;

- $C_{i}$ is maximal in the sense that if $C_{i}$ is contained in a set $E$, then $E$ does not satisfy the first or the second condition.

- The $C_{i}$ are disjoint and their complementary is a set of null measure.

The three main steps involved in the analysis are:

- Computation of a subset of the joint space (resp. workspace) where the number of solutions changes: the Discriminant Variety [5].

- Description of the complementary of the discriminant variety in connected cells: the Generic Cylindrical Algebraic Decomposition.

- Connecting the cells belonging to the same connected component in the counterpart of the discriminant variety: interval comparisons.

From a general point of view, the discriminant variety is defined for any system of polynomial equations and inequalities. The union of the Discriminant variety and of the cells thus define a partition of the considered space. Let $p_{1}, \ldots, p_{1}$ and $q_{1}, \ldots, q_{l}$ be polynomials with rational coefficients depending on the unknowns $X_{1}, \ldots, X_{n}$, and on the parameters $U_{1}, \ldots, U_{d}$. Let us consider the constructible set:

$$
\mathscr{C}=\left\{\begin{array}{l}
\mathbf{v} \in \mathbb{C}^{n+d}, p_{1}(\mathbf{v})=0, \ldots, p_{m}(\mathbf{v})=0, \\
q_{1}(\mathbf{v}) \neq 0, \ldots, q_{l}(\mathbf{v}) \neq 0
\end{array}\right\}
$$

If we assume that $\mathscr{C}$ is a finite number of points for almost all the parameter values, a discriminant variety $V_{D}$ of $\mathscr{C}$ is a variety in the parameter space $\mathbb{C}^{d}$ such that, over each connected open set $\mathscr{U}$ satisfying $\mathscr{U} \cap V_{D}=\emptyset, \mathscr{C}$ defines an analytic covering. In particular, the number of points of $\mathscr{C}$ over any point of $\mathscr{U}$ is constant.

Let us now consider the following semi-algebraic set:

$$
\mathscr{S}=\left\{\begin{array}{l}
\mathbf{v} \in \mathbb{C}^{n+d}, p_{1}(\mathbf{v})=0, \ldots, \\
p_{m}(\mathbf{v})=0, q_{1}(\mathbf{v}) \geq 0, \ldots, q_{l}(\mathbf{v}) \geq 0
\end{array}\right\}
$$

If we assume that $\mathscr{S}$ has a finite number of solutions over at least one real point that does not belong to $V_{D}$, then $V_{D} \cap \mathbb{R}^{d}$

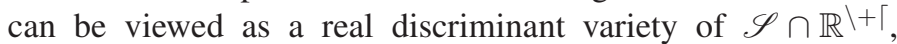
with the same property: over each open set $\mathscr{U} \subset \mathbb{R}^{\lceil}$such that $\mathscr{U} \cap \mathscr{V}_{D} \cap \mathbb{R}^{d}=\emptyset, \mathscr{C}$ defines an analytic covering. In particular the number of real points of $\mathscr{S}$ over any point of $\mathscr{U}$ is constant. Discriminant varieties can be computed using basic and well known tools from computer algebra such as Groebner bases (see [15], Chapter 3) and a full package computing such objects 
in a general framework is available in Maple software through the RootFinding[Parametric] package.

The cylindrical algebraic decomposition (CAD) implemented in the SIROPA library has been used to compute the aspects into a set of cells where algebraic equations define its boundaries and a sample point in each one [5] for the 2PRRRPR parallel robot. For example, the CAD can provide a formal decomposition of the joint space in cells where the polynomials $\operatorname{det}(\mathbf{A})$ and $\operatorname{det}(\mathbf{B})$ have a constant sign and the number for the DKP is constant [16].

\section{WORKSPACE AND JOINT SPACE ANALYSIS Joint Space Analysis}

The joint space analysis allows the characterization of the regions where the number of real solutions for the direct kinematic model is constant. Using CAD, we can do this study on sections of the joint space. The calculation for the full joint space is possible, but the number of cells obtained is too large for the display capabilities of Maple. Without taking into account the notion of operation mode, Figure 4 depicts the regions with 4, 8, 12 or 16 solutions for the DKP. The maximum number for the DKP of each operation mode is 8 .

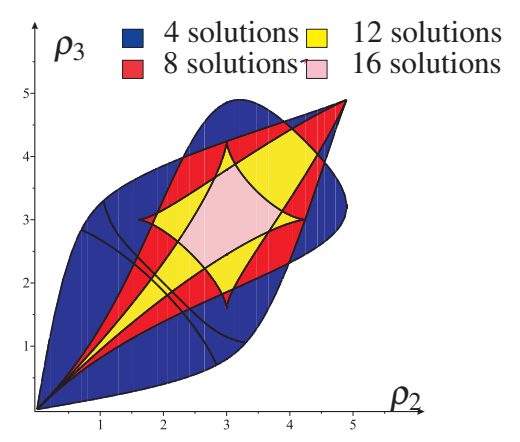

FIGURE 4. Slice of the joint space for $\rho_{1}=3$ and the number of solution for DKP

Figures 5 and 6 show several slices of the joint space for $O M^{1}$ and $O M^{2}$, where the DKP admits four and eight real solutions in the blue and red region respectively.

Cuspidal configurations are associated with second-order degeneracies that appear for triply coalesced configurations. These configurations play an important role in the path planning because they are directly linked to the non-singular assembly mode changing trajectories [6,17-19]. A state of the art for the computation of the cusp points is given in [20]. Here we make use of the Jacobian criterion on the singular locus to extract the points of multiplicity greater than or equal to three. Figures 5 and 6 show that the number of cusp points is changing according to the value of $\rho_{1}$. Figure 7 depicts the locus of the cusp points in the joint space. These curves are obtained as the intersection of 11 surfaces for $O M^{1}$ and 8 surfaces for $O M^{2}$. However, for the 3-RPS parallel robot is very difficult to remove the multiplicity greater than two by using the saturation method as introduced in [21].

\section{Workspace Analysis}

The workspace of the robot is a cylinder in the projection space $\left(z, q_{2}, q_{3}\right)$ if there are no joint limits on the actuated joints.

The workspace analysis can be done by dividing it into a set of aspects. The notion of aspect, previously defined in [22] for serial robots and in [8] for parallel robot with one operation mode can be extended for a parallel robot with several operation modes such that:

- $W A_{i}^{j} \subset W_{O M^{j}}$

- $W A_{i}^{j}$ is connected

- $\forall X \in W A_{i}^{j}, \operatorname{det}(\mathbf{A}) \neq 0$ and $\operatorname{det}(\mathbf{B}) \neq 0$

In other words, an aspect $W A_{i}^{j}$ is the largest connected region without any singularity of the $O M^{j}$.

The analysis of the workspace is done in the projection space $\left(z, q_{2}, q_{3}\right)$. We found out four aspects as shown in Figure 8 .

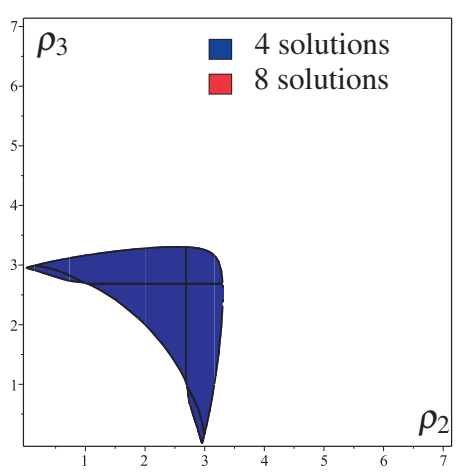

(a)

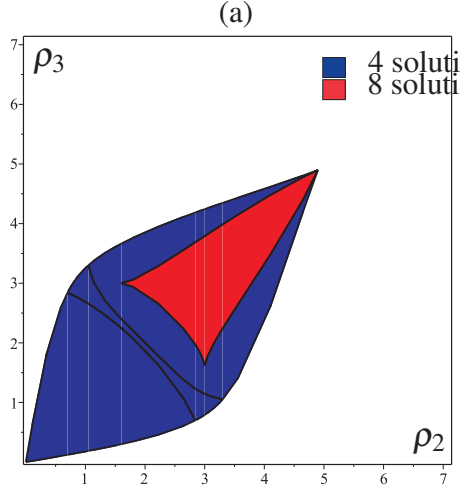

(c)

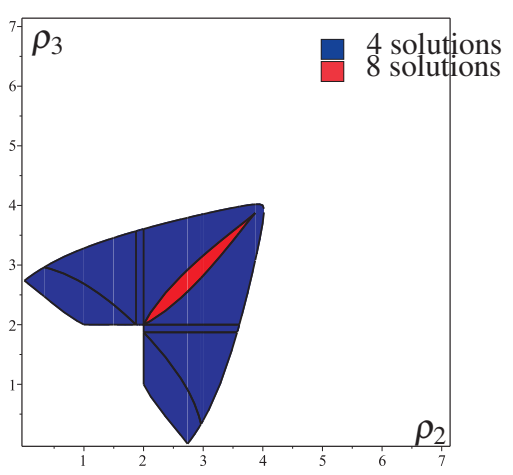

(b)

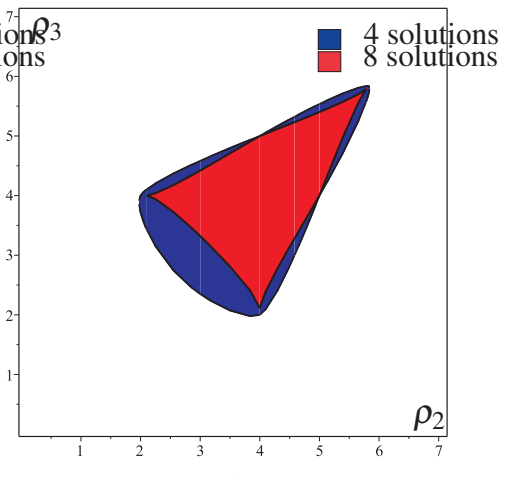

(d)
FIGURE 5. Slice of the joint space for $O M^{1}$ for $\rho_{1}=1$ (a), $\rho_{1}=2$ (b), $\rho_{1}=3$ (c) and $\rho_{1}=4$ (d) and the number of solution for DKP 